\title{
Behavioral responses to visual overstimulation in the cockroach Periplaneta americana $\mathbf{L}$.
}

\author{
Marianna Zhukovskaya ${ }^{1}$, Ekaterina Novikova ${ }^{1}$, Paulus Saari ${ }^{2}$, Roman V. Frolov ${ }^{2}$
}

${ }^{1}$ Sechenov Institute of Evolutionary Physiology and Biochemistry, Russian Academy of Sciences,

44 Thorez Pr., Saint Petersburg 194223, Russia

${ }^{2}$ Faculty of Science, Nano and Molecular Materials Research Unit, University of Oulu, P.O.Box 3000, 90014 Oulun Yliopisto, Finland.

Corresponding author: Roman V. Frolov², Faculty of Science, Nano and Molecular Materials Research Unit, University of Oulu, P.O.Box 3000, 90014 Oulun Yliopisto, Finland, e-mail: rvfrolov@oulu.fi. ORCID: 0000-0002-7431-5297. 


\begin{abstract}
In the visual systems of insects, different types of photoreceptors contribute to specialized visual channels that mediate distinct functions and behaviors. Large compound eyes of Periplaneta americana contain photoreceptors of two spectral classes, broadband green-sensitive photoreceptors and narrowband UV-sensitive photoreceptors. Here, we investigated how visual stimulation by UV and green light affects locomotor, resting, and grooming behaviors in $P$. americana under conditions when light avoidance is not possible. We show that green but not UV light stimulates locomotor activity, inducing paradoxical positive masking. Duration of resting and grooming decreased with increasing light intensity, consistent with development of behavioral stress in response to visual overstimulation. A reaction of full immobility is described under UV light and at higher intensities of green light, with relative periods of immobility and grooming strongly negatively correlated. Low-intensity UV was more effective than low-intensity green light in suppressing grooming and inducing immobility. Our results suggest that locomotor activity in $P$. americana is mainly regulated by green-sensitive photoreceptors, and that dim UV light can trigger behavioral immobility, whereas both wavelengths induce stress-like reactions at high intensities. Considering the intrinsic UV sensitivity of green-sensitive photoreceptors, the contrasting behavioral responses indicate antagonistic interactions between UV and green visual channels.
\end{abstract}

Keywords: Periplaneta americana, vision, locomotor behavior, grooming, immobility. 


\section{Introduction}

The American cockroach, Periplaneta americana, is a nocturnal insect with large compound eyes. Under normal conditions, cockroaches are active during the night, engaging in exploration, feeding, and mating (Lipton and Sutherland 1970a; Lipton and Sutherland 1970b; Seelinger 1984), while avoiding illuminated areas (Kelly and Mote 1990). One of the most salient behavioral features of $P$. americana is its escape reaction in response to sudden illumination: when a dark-adapted cockroach is exposed to light, it immediately bolts toward the nearest dark shelter. Such reactions to light depend on its intensity and wavelength (Kelly and Mote 1990) and are principally based on light input through the compound eyes (Okada and Toh 1998).

The visual system of $P$. americana consists of several sub-systems or channels morphologically based on the compound eyes and ocelli. The apposition-type compound eye contains: a powerful and highly sensitive broadband motion detection "scotopic" channel predominantly mediated by green-sensitive photoreceptors (Honkanen et al. 2014); a color vision channel as evidenced by the presence of ultraviolet/green color opponent neurons in the optic lobe (Kelly and Mote 1990); and a polarization-sensitive channel (Butler and Horridge 1973; Kelly and Mote 1990). The simple eyes, or ocelli, that appear to be involved in a myriad functions that vary from species to species, ranging from motion detection to circadian rhythm and flight control regulation (Parry 1947), are also present in P. americana, where they modulate escape reactions in very dim light (Okada and Toh 1998). This role of the ocelli is corroborated by the morphological evidence of enormous spatial summation taking place in the ocellar plexus, where the axons of all ten thousand ocellar photoreceptors converge on just four second-order neurons (Mizunami 1995).

Spectroscopic and electrophysiological studies revealed that ommatidia in the compound eye of $P$. americana contain rhabdomeres of two spectral types, with maximal sensitivity to UV (365 $\mathrm{nm}$ ) or green (ca. $510 \mathrm{~nm}$ ) light (Mote and Goldsmith 1970). According to intracellular recording and patch-clamp studies, green-sensitive photoreceptors are at least ten times more numerous than UV photoreceptors (Heimonen et al. 2006). There is no apparent segregation of photoreceptor types into specialized regions or zones of the eye, as it is observed in other nocturnal insects (Frolov et al. 2014). Instead, UV- and green-sensitive photoreceptors appear to be interspersed in the ommatidia, although it is possible that densities of UV and green photoreceptors are dissimilar in different parts of the eye. Recent retinal transcriptome analysis with consecutive RNA interference experiments discovered the presence of three opsins in the compound eye retina, one UV and two green ones, with one green opsin more abundant than the other by several orders of magnitude (French et al. 2015). 
The escape reaction in response to illumination that exceeds a certain intensity threshold can be a manifestation of stress in an animal, which is suddenly exposed to dramatic sensory overstimulation. However, despite extensive observations of behaviors associated with overstimulation of the visual system in P. americana and similar nocturnal insect species (Godden and Goldsmith 1972; Shi et al. 2017; Shimoda and Honda 2013), questions remain about relative contributions of different visual channels to visual overstimulation-induced reactions, and to specific behavioral reactions associated with stress. Grooming, a complex, often stereotypic process of cleaning body surfaces and sensory appendages, which has been extensively studied in both vertebrates and insects (for review see (Hlavac 1975; Smith and Valentine 1985)), is one such behavior that changes in stressed animals (Kalueff et al. 2016; Tinbergen 1951; Zhukovskaya et al. 2013).

In this work, we characterized behavioral responses in $P$. americana to UV and green light over a wide range of light intensities and describe a novel immobility reaction, which develops in progressively brighter light. As green-sensitive photoreceptors are characterized by high sensitivity to UV, our results strongly suggest the presence of antagonistic interactions between visual channels processing responses of UV- and green-sensitive photoreceptors in the higher visual centers of $P$. americana nervous system.

\section{Materials and Methods}

\section{Animals and the experimental setup}

American cockroaches P. americana (L.) were reared locally (St. Petersburg, Russia) and maintained in reversed L/D $12: 12 \mathrm{~h}$ illumination conditions at temperatures from 24 to $28^{\circ} \mathrm{C}$. A fluorescent lamp emitting a substantial amount of UV light was used as a light source during the day phase.

Behavioral experiments were performed in the following way. The setup consisted of three connected compartments (Fig. 1a), two of which, a constant dark "shelter" and a L/D 12:12 h illuminated "habitat" (Fig. 1b) supplied with food and water ad lib, were available to animals all the time, while the third one, a detachable testing chamber, was available only during testing. The testing chamber was equipped with a side panel for illumination with dark red light (Fig. 1B); two LEDs, green and UV, and a VSC-756 USB high-resolution monochrome video camera (EVS, Russia) $25 \mathrm{~cm}$ above the floor; and a moist air inlet. Narrow-band 1W 019595 ARPL-1W-EPL UV400 (UV, spectral peak at $400 \mathrm{~nm}$ ) and 1W Green ARPL-Star (green, spectral peak at $530 \mathrm{~nm}$ ) LEDs were used in experiments (Fig. 1c). LED emission spectra were measured using an Ocean Optics spectrometer (Ocean Optics, Dunedin, FL, USA). Use of forced wet air ventilation was 
essential to prevent development of dehydration-induced behavioral changes during the prolonged recordings, as well as odor accumulation. The flow of air was ca. $100 \mathrm{ml} \mathrm{min}^{-1}$. Adult cockroaches ( up to 2 weeks after the final molt) were placed in the living compartment two weeks prior to the experiments.

\section{Experimental procedure}

Experiments were performed in the beginning of the night, when cockroaches are most behaviourally active. During each experiment, a single cockroach was allowed to enter the testing chamber and explore it in red light for $10 \mathrm{~min}$ (Fig. 1d). After that, a 30-min video recording was performed. This recording session (henceforth "session 1" or S1) was followed by another $10 \mathrm{~min}$ period in red light without recording, after which a $30 \mathrm{~min}$ light exposure testing session was started (henceforth “session 2" or S2). Throughout the text ( $n$ ) indicates sample size.

In control experiments $(n=11)$, during session 2, the animal was exposed to the same dark red light as during session 1. In light exposure experiments, the chamber was illuminated with one intensity of green or UV light. Three UV and three green light intensities were used in the experiments. When the light intensities were measured with a digital optical power meter Thorlabs PM100D equipped with a photodiode sensor S121C (Thorlabs Inc., Newton, NJ, USA) in the middle of the floor of the testing chamber, the photon fluxes were $2.8 \cdot 10^{10}, 8.5 \cdot 10^{12}$, and $2.4 \cdot 10^{14}$ photons s$~^{-1} \mathrm{~cm}^{-2}$ for the UV LED, and $4.1 \cdot 10^{12}, 9.7 \cdot 10^{13}$, and $1.1 \cdot 10^{15}$ photons $\mathrm{s}^{-1} \mathrm{~cm}^{-2}$ for the green LED. Each cockroach was used in experiments only once. Between the experiments, the test chamber was cleaned thoroughly. The numbers of experiments for the six different conditions were respectively: $12,10,13,12,11$, and 14 .

\section{Intracellular recordings}

In vivo intracellular single-electrode recordings and light stimulation were performed as described previously (Saari et al. 2017). In brief, microelectrodes were manufactured from borosilicate glass (Harvard Apparatus, Holliston, MA, USA) with a laser puller (P-2000; Sutter Instrument Novato, CA, USA) and filled with $2 \mathrm{M} \mathrm{KCl}$ solution, $\mathrm{pH} 6.84$, to the final resistance of 100-150 M 2 . The reference electrode was placed through an antenna. Signals were recorded with an intracellular amplifier (SEC-05L; npi electronic, Tamm, Germany). For light stimulation, a computer-controlled custom voltage-to-current LED driver was used to drive 14 LEDs (Roithner Laser Technik, Austria), with narrow-band emission peaks ranging from 355 to $625 \mathrm{~nm}(355,385,400,435,450$, $462,490,505,525,545,572,594,612,625 \mathrm{~nm}$ ), in combination with a series of neutral density filters (Kodak, New York, NY, USA).

\section{Data analysis and statistics}


Analysis of video files was performed manually frame by frame using VirtualDub (free software by Avery Lee). During analysis of grooming, a grooming sequence was defined as a sequential cleaning of several body parts without interruption by other behaviours. A grooming sequence included some of the following elements: cleaning of the antennal flagellum with the mouthparts; scraping of the antennal base with a foreleg; cleaning of the forelegs, mid legs and hind legs with the mouthparts; and cleaning of the cerci and genitalia with the hind legs. Locomotion was measured by counting the number of quadrants the cockroach walked across during the recording sessions (Fig. 1a).

During statistical data analysis, the Shapiro-Wilk normality test was applied to data samples to determine if they could be analyzed using parametric statistical methods. If data in some samples describing a certain behaviour type failed the normality test, or passed only marginally for a minority of experimental groups, such data were presented using medians and interquartile ranges (25\% quartile:75\% quartile), and compared using the Wilcoxon signed-rank test (WSRT) for paired results or the Mann-Whitney U test (MWUT) for independent samples. Such data are presented in the figures using box plots; the box plots show medians, interquartile ranges (error bars), and 5:95\% outliers. The data that passed the normality test were presented as means \pm standard deviation, and compared using Student's $t$-test as indicated. Pearson Correlation Coefficient $(r)$ was used in the analysis of correlations. In the figures, $\left(^{*}\right)$ stands for $0.05<P<0.001$, and $(* *)$ for $P<0.001$.

\section{Results}

\section{Behavioral experiments}

Analysis of video recordings of cockroach behavior in the testing chamber during S1 and S2 allowed us to define and quantify four distinct behaviors: locomotion, grooming, resting behavior, and a novel immobility reaction. Resting was defined as a behavior without visible leg movement, which, however, was always accompanied by some movement of the antennae, or the mouthparts. The immobility reaction is defined here as an inactive, immobile state resembling resting but without any movements. Immobility could occur after resting or grooming, and while its onset was straightforward to determine during resting, transition from grooming to immobility was more difficult to define. Grooming of the mouthparts was generally impossible to resolve in our video recordings and if immobility occurred after grooming, in order to account for this uncertainty, the duration of such an immobility episode was reduced by one minute.

Figure 2 summarizes changes in locomotion. In control experiments, the median number of quadrants visited decreased from 92 (63:112) during $\mathrm{S} 1$ to 30 (14:51) during $\mathrm{S} 2(P=0.0036, n=$ 11 , WSRT), which obviously reflects a decrease in the exploratory activity due to adaptation of the 
cockroach to its new environment (Fig. 2a). Similar decreases in locomotor activity were observed in the animals exposed to UV light at all three intensities, $\operatorname{dim}(P=0.0024, n=12, \mathrm{WSRT})$, intermediate $(P=0.0074, n=10$, WSRT), and bright UV $(P=0.004, n=13$, WSRT, Fig. 2a). In contrast, cockroaches exposed to green light showed notably smaller, statistically insignificant decreases in the number of quadrants visited during S2 (Fig. 2a). The relative changes in locomotion are shown in Figure 2b. Since no intensity-dependence of the locomotor activity decrease was observed, data within experimental green and UV groups were pooled together to facilitate statistical analysis. Figure $2 \mathrm{c}$ shows that under green light cockroaches moved significantly more than in control ( $P=0.004$, MWUT, $n=37$ and 11 , respectively) or in UV light $(P<0.001$, MWUT, $n=37$ and 35, respectively).

Figure 3 specifies how long the cockroaches were engaged in each particular behavior under different conditions. The pie charts were generated by summing up the durations of each behavior for the entire experimental group. Since locomotion was quantified in terms of quadrants visited (Fig. 2), while the periods of other behaviors were measured directly, the period of locomotion was estimated by subtracting the durations of three other behaviors from the total time of experimental observation. During S1 cockroaches were predominantly engaged in locomotion (85 to $93 \%$ of observation time). Remaining time was divided between grooming, and, to a much smaller extent, resting. No incidents of immobility were observed. During S2, locomotion was reduced notably in the control group, the time share of resting behavior increased, and cockroaches spent $2 \%$ of time in the completely immobilized state.

In the experiments involving illumination with green light, the behavior durations changed dramatically. In the dimmest green light, resting behavior was virtually abolished due to increased locomotion, while grooming duration increased slightly. At the next, intermediate green light level, 3 out of 11 animals exhibited episodes of immobility lasting from 16 to $882 \mathrm{~s}$, which accounted for $5 \%$ of the total observation time (Fig. 3). In the brightest green light, 9 out of 14 cockroaches displayed episodes of immobility, altogether accounting for $16 \%$ of the recording period.

Under UV light, the time spent for locomotion was reduced to the same level as in control, but instead of resting and grooming, cockroaches were in the immobility state more than half of the locomotion-free time (Fig. 3). Specifically, 3 out of 11 cockroaches in dim UV light, 8 out of 9 at the intermediate UV intensity, and 9 out of 13 animals in bright UV light displayed immobility reactions of varying duration.

Figure 4 illustrates changes in the grooming behavior. Grooming sequences were characterized by variable durations, depending on the number of appendages cleaned on each occasion. We restricted our analysis to determining: the share of time spent for grooming for each 
experimental group (Fig. 3); the number and average duration of grooming sequences (Fig. 4a, c); and the duration of two major and unambiguously definable grooming events, cleaning of the antennal flagella, and mid and hind legs combined (Fig. 4e).

In dim green light, the total grooming duration during S2 remained the same as in control. However, it decreased notably in brighter green and at all three intensities of UV light, due to a decrease both in the number of grooming sequences and in their average duration (Fig. 4a-c). A strong negative correlation was found between the amounts of time cockroaches spent grooming and in immobile state (Fig. 4d). The Pearson's $r$ was $-0.81(n=7, P=0.03)$. To investigate how illumination altered the duration of specific grooming events, we compared the relative durations of antennal grooming and grooming of mid and hind legs during S2 (Fig. 4e). Whereas the antennal grooming was largely unaffected, exposure to progressively bright light led to reduction of leg cleaning.

Figure 5 illustrates dependencies of changes in the four behaviors on the actual photon fluxes during S2. Our data indicate that UV light is extremely effective at suppressing grooming and inducing immobility (Fig. 5a, b). Differential effects on locomotion and resting time shares were less prominent (Fig. 5c, d).

\section{Intracellular recordings}

Here we found contrasting behavioral responses to illumination by green and UV light. However, previous electrophysiological studies of visual pigments in insects established the presence of secondary higher-frequency absorbance bands, including the most physiologically relevant betaband centering around $400 \mathrm{~nm}$ (Stavenga et al. 1993). Therefore, it was necessary to see if greensensitive photoreceptors in the compound eyes of $P$. americana exhibit any substantial intrinsic sensitivity to UV light.

Figures $6 \mathrm{a}$ and $\mathrm{b}$ demonstrate typical action spectra of UV- and green-sensitive photoreceptors. As expected, broad-band photoreceptors had a secondary peak at $385 \mathrm{~nm}$, indicating a substantial sensitivity to UV (Fig. 6b, c). When the actual spectral sensitivities of the two photoreceptors were calculated using a previously published data sample (Saari et al. 2017), for the green-sensitive photoreceptor the ratio of UV to green peaks was reduced to 0.35 . The UVsensitive photoreceptor was characterized by narrow sensitivity to UV light with a peak at $385 \mathrm{~nm}$.

\section{Discussion}

In this work we characterized the effects of narrow band UV and green light over a hundred-fold intensity range on several specific behaviors of $P$. americana and described a new type of behavior, 
a sleep-like immobility reaction. Although similar experiments have been performed previously (Kelly and Mote 1990), they were focused on the analysis of light avoidance behavior, whereas here we specifically investigated how illumination alters individual behavior under conditions when avoidance is impossible. Our research builds upon previous electrophysiological studies in $P$. americana, which found only two spectral types of photoreceptors in the compound eyes, UV- and green-sensitive ones (Heimonen et al. 2006; Kelly and Mote 1990) and determined that physiological avoidance reactions are principally mediated by the compound eyes (Okada and Toh 1998). It should be noted that we did not attempt to evaluate effects of illumination on other behaviors, such as feeding or mating, or any other social interactions among cockroaches.

The main individual behaviors of $P$. americana are locomotion, resting, and grooming. Locomotion is an explorative behavior, whereas resting and grooming are sedentary behaviors. Importantly, studies in both vertebrates and invertebrates showed that the duration of grooming decreases and its patterns change when animals are stressed (Kalueff et al. 2016; Tinbergen 1951; Zhukovskaya et al. 2013), which makes the duration of grooming a useful proxy for stress.

In natural environments small nocturnal animals rarely or never experience exposure to daylight levels of illumination, as they leave shelter in the dark and reenter it before sunrise. Here we described immobility as a behavioral reaction associated with an abnormal visual stimulation. Is there a sufficient experimental justification for distinguishing immobility from other behaviors, such as resting? Our findings suggest that immobility appears to be a distinct reaction. It was different from the normal resting since the cockroach became completely immobile. The relative duration of immobility was short under dim red or dim green light, and increased with green light intensity. However, immobility manifested prominently in dim UV light, when the photon flux was approximately two orders of magnitude lower than in dim green light.

Immobility observed in our experiments seems to be different from a "freezing" reaction, which could be triggered in insects with sudden illumination and could be seen as an adaptive behaviour during the initial overstimulation blindness, because an immobile target is less likely to be spotted by predators than a moving one. In our experiments immobility episodes were not associated with a sudden change in illumination but rather happened spontaneously and were also registered during S2 in control experiments under dark red illumination.

Although immobility appears to be a sleep-like condition, we did not attempt to characterize and compare the natural sleep with immobility in this work. However, the light-induced immobility is clearly different from thanatosis, a well-characterized tonic immobility behavior observed in a wide range of species across the animal kingdom, including crickets and stick insects (Godden and Goldsmith 1972; Nishino 2004): in contrast to thanatosis, the immobility observed here did not 
develop suddenly in response to the onset of light, but rather gradually; also, it ended and recurred spontaneously.

Immobility appears to be different from a behavior called "learned helplessness". Essentially, the behavior can be defined as tolerating a stressful situation without attempting to escape it, while the escape is possible. It develops in an animal, which was previously subjected to the same distressful factor but in a situation when avoidance was not possible (Eisenstein and Carlson 1997). The phenomenon is widespread and can be induced in vertebrates and invertebrates alike and doesn't require brain for establishing. However, as we report here, immobility reaction occurred without prior conditioning, its episodes ended and recurred spontaneously, and it occurred on few instances in control S2. In one study on learned helplessness in P. americana, no detailed description of cockroach behavior was provided (Brown and Stroup 1988), making it impossible to compare learned helplessness with the reaction of immobility.

Overall, although immobility seems to be different from resting, "freezing", and "learned helplessness" behaviors, more research is needed to properly establish its characteristics.

How do the overall patterns of the four behaviors change with illumination? We showed that locomotor activity of cockroaches decreased dramatically in control from S1 to S2, indicating that by the time of S2 cockroaches had more or less completed exploration of the testing compartment. In green light, however, cockroaches became agitated again and significantly increased their activity, possibly looking for escape from the test chamber. This response to light falls under the definition of the paradoxical positive masking as "an increase in activity after an increase in illumination in a nocturnal animal" by N. Mrosovsky (Mrosovsky 1999).

Whereas no evidence of stress was found under dim green light, the total grooming time, the number of grooming sequences and their average durations decreased in green light of intermediate and bright intensities (Fig. 4), suggesting growing stress. Close examination of the grooming sequence revealed that grooming duration decreased largely by reduced grooming of mid and hind legs, which usually takes place at the end of the grooming sequence. Increasing UV intensity caused similar changes in grooming, i.e. decrease in the number of grooming sequences and their durations, with shorter leg grooming, indicating increased stress level (Fig. 4). Still, the question remains why dim green light caused locomotor agitation. One possibility could be that selective stimulation of the green visual channel represents an abnormal situation since UV is always present under natural conditions (see below).

At the same time, the total duration of immobility episodes increased in an intensitydependent manner, from $2 \%$ in control and dim green to $16 \%$ in bright green light. In comparison, 
locomotor activity did not differ from control under UV light at all three intensities, which was a counterintuitive finding since UV illumination was previously shown to be more effective in evoking an escape reaction (Kelly and Mote 1990). It is of particular importance that exposure to the lowest UV intensity, roughly corresponding to UV fluxes at environmental irradiance approximately one order of magnitude higher than irradiance from the moon (Cronin et al. 2014), produced a decrease in grooming and an increase in immobility periods, which was similar to that induced by bright green light, at four orders of magnitude higher photon flux (Fig. 5a, b). This behavior might be classified as negative masking (Mrosovsky 1999). This disparity gets even more salient when one notices that UV photoreceptors are underrepresented in the compound eyes of $P$. americana. Because environmental levels of UV radiation depend less on weather conditions than visible light (Cronin et al. 2014), it is possible that the UV-sensitive visual channel has evolved as the main circadian switch controlling P. americana behavior.

The strikingly different behavioral reactions to UV and green illumination are particularly interesting in light of intrinsic sensitivity of green-sensitive photoreceptors to UV (Fig. 6b, c). It follows that when cockroaches are irradiated by green light, only green-sensitive photoreceptors respond. When, on the other hand, UV light is used, both UV- and green-sensitive photoreceptors activate. Therefore, the immobility behavior is triggered when both UV- and green-sensitive photoreceptor are active, whereas increased locomotion is observed when only green-sensitive photoreceptors are active. Such a situation implies antagonistic interactions between UV and green visual channels, with the physiological outcome depending on the strength of stimulation via each channel. Indeed, the presence of inhibitory interactions in the medulla between interneurons receiving innervations from specific receptor types was demonstrated in the previous study (Kelly and Mote 1990).

In conclusion, we have assessed the influences of the UV and green visual channels on individual behavior in $P$. americana and found substantially different patterns of responses. Our results suggest that excitation of the green but not UV channel stimulates locomotor behavior, while low-intensity UV stimulation triggers sleep-like behaviors. Thus our results suggest that UV and green visual channels indeed mediate different visual functions, that bright illumination causes stress independent of wavelength, and that the two channels are mutually antagonistic.

\section{Acknowledgements}

The authors thank the following: Dr. Victor I. Govardovskii for design of the lighting devices, measuring their emission spectra, and discussions; Alexander Miltsin for assembling and installing the lighting devices and their controller; and Dr. Roman V. Cherbunin for lighting source 
calibration The study was supported by Russian Federation State budget grant \# 01201351571 (2013-2017).

\section{References}

Brown GE, Stroup K (1988) Learned helplessness in the cockroach (Periplaneta americana) Behavioral and Neural Biology 50:246-250 doi:http://dx.doi.org/10.1016/S0163-1047(88)90921-1

Butler R, Horridge GA (1973) The electrophysiology of the retina of Periplaneta americana L. Journal of comparative physiology 83:279-288 doi:10.1007/bf00693679

Cronin TW, Johnsen S, Marshall NJ, Warrant EJ (2014) Light and the Optical Environment. In: Visual Ecology. Princeton University Press, pp 10-36

Eisenstein EM, Carlson AD (1997) A comparative approach to the behavior called 'learned helplessness' Behavioural Brain Research 86:149-160 doi:http://dx.doi.org/10.1016/S0166-4328(96)02260-7

French AS, Meisner S, Liu H, Weckstrom M, Torkkeli PH (2015) Transcriptome analysis and RNA interference of cockroach phototransduction indicate three opsins and suggest a major role for TRPL channels Front Physiol 6:207 doi:10.3389/fphys.2015.00207

Frolov RV, Immonen EV, Weckström M (2014) Performance of blue- and green-sensitive photoreceptors of the cricket Gryllus bimaculatus J Comp Physiol A Neuroethol Sens Neural Behav Physiol 200:209219 doi:10.1007/s00359-013-0879-6

Godden D, Goldsmith TH (1972) Photoinhibition of arousal in the stick insect Carausius Zeitschrift für vergleichende Physiologie 76:135-145 doi:10.1007/bf00455958

Heimonen K, Salmela I, Kontiokari P, Weckström M (2006) Large functional variability in cockroach photoreceptors: optimization to low light levels J Neurosci 26:13454-13462 doi:26/52/13454 [pii]

10.1523/JNEUROSCI.3767-06.2006

Hlavac TF (1975) Grooming Systems of Insects: Structure, Mechanics1 Annals of the Entomological Society of America 68:823-826 doi:10.1093/aesa/68.5.823

Honkanen A, Takalo J, Heimonen K, Vähäsöyrinki M, Weckström M (2014) Cockroach optomotor responses below single photon level The Journal of experimental biology 217:4262-4268 doi:10.1242/jeb.112425

Kalueff AV, Stewart AM, Song C, Berridge KC, Graybiel AM, Fentress JC (2016) Neurobiology of rodent selfgrooming and its value for translational neuroscience Nat Rev Neurosci 17:45-59 doi:10.1038/nrn.2015.8

http://www.nature.com/nrn/journal/v17/n1/abs/nrn.2015.8.html\#supplementary-information

Kelly KM, Mote MI (1990) Electrophysiology and anatomy of medulla interneurons in the optic lobe of the cockroach, Periplaneta americana Journal of Comparative Physiology A 167:745-756 doi:10.1007/bf00189765

Lipton GR, Sutherland DJ (1970a) Activity rhythms in the American cockroach, Periplaneta americana Journal of Insect Physiology 16:1555-1566 doi:http://dx.doi.org/10.1016/0022-1910(70)90254-4

Lipton GR, Sutherland DJ (1970b) Feeding rhythms in the American cockroach, Periplaneta americana Journal of Insect Physiology 16:1757-1767 doi:http://dx.doi.org/10.1016/0022-1910(70)90274-X

Mizunami M (1995) Functional diversity of neural organization in insect ocellar systems Vision Research 35:443-452 doi:http://dx.doi.org/10.1016/0042-6989(94)00192-0

Mote MI, Goldsmith TH (1970) Spectral sensitivities of color receptors in the compound eye of the cockroach Periplaneta Journal of Experimental Zoology 173:137-145 doi:10.1002/jez.1401730203

Mrosovsky N (1999) Masking: history, definitions, and measurement Chronobiology international 16:415429

Nishino H (2004) Motor output characterizing thanatosis in the cricket Gryllus bimaculatus Journal of Experimental Biology 207:3899-3915 doi:10.1242/jeb.01220 
Okada J, Toh Y (1998) Shade Response in the Escape Behavior of the Cockroach, Periplaneta americana Zoological Science 15:831-835 doi:10.2108/zsj.15.831

Parry DA (1947) The function of the insect ocellus The Journal of experimental biology 24:211-219

Saari P, French AS, Torkkeli PH, Liu H, Immonen EV, Frolov RV (2017) Distinct roles of light-activated channels TRP and TRPL in photoreceptors of Periplaneta americana The Journal of general physiology 149:455-464 doi:10.1085/jgp.201611737

Seelinger G (1984) Sex-specific Activity Patterns in Periplaneta americana and their Relation to Mate-finding Zeitschrift für Tierpsychologie 65:309-326 doi:10.1111/j.1439-0310.1984.tb00107.x

Shi L, Vasseur L, Huang H, Zeng Z, Hu G, Liu X, You M (2017) Adult Tea Green Leafhoppers, Empoasca onukii (Matsuda), Change Behaviors under Varying Light Conditions PloS one 12:e0168439 doi:10.1371/journal.pone.0168439

Shimoda M, Honda K-i (2013) Insect reactions to light and its applications to pest management Applied Entomology and Zoology 48:413-421 doi:10.1007/s13355-013-0219-x

Smith BJB, Valentine BD (1985) Phylogenetic Implications of Grooming Behavior in Cockroaches (Insecta: Blattaria) Psyche 92:369-385 doi:10.1155/1985/81520

Stavenga DG, Smits RP, Hoenders BJ (1993) Simple exponential functions describing the absorbance bands of visual pigment spectra Vision Res 33:1011-1017

Tinbergen N (1951) The study of instinct. Clarendon Press, Oxford

Zhukovskaya M, Yanagawa A, Forschler B (2013) Grooming Behavior as a Mechanism of Insect Disease Defense Insects 4:609

\section{Legends to Figures}

Fig. 1 The experimental setup and methodology. a The experimental setup consisted of three chambers: a small, constantly dark shelter, a larger living compartment containing water and food ad lib, and an adjacent testing chamber accessible only during the actual experiment. The test chamber was equipped with green and UV LEDs, and a dark red side panel (see photograph in panel B); it also had a moist air inlet and a camera in the middle of the ceiling. $\mathbf{b}$ A photograph of a cockroach in the testing chamber during an experiment in dark red light. c Spectra of UV and green LEDs used in experiments. d Schematic description of the experimental methodology. During S1, cockroach behavior was recorded for $30 \mathrm{~min}$ in dark red light, preceded and followed by $10 \mathrm{~min}$ periods without recording. During S2, in control experiments, only dark red light of the same intensity as during S1 was used; in light exposure experiments, cockroaches were presented with 30 min of steady green or UV light of three different intensities, so that each individual cockroach was exposed to one intensity only

Fig. 2 Changes in locomotor activity under green and UV light. a Box plots describe the median numbers of quadrants of the testing chamber floor walked over by a cockroach during S1 (left column in each pair) or S2 (right column) experiments; light intensities are specified in Methods, with the lowest intensity denoted here as "dim", intermediate one as "med.", and the highest one as "bright"; the number of animals in each experimental group varied from 11 to 14 (see Results); grey dashed lines separate experimental groups. b The relative numbers of quadrants visited during S2; values were obtained by dividing the numbers of quadrants visited during S2 by those in S1 for 
each animal. c Since the differences in locomotor activity between three intensities for each LED were insignificant, all green and UV exposure data were pooled together and compared; locomotor activity increased significantly in green but not in UV light

Fig. 3 Contributions of different behaviors change with light intensity. Pie charts show the relative periods cockroaches engage in each of four distinct behaviors (locomotion, resting, grooming, and immobility) during S1 and S2

Fig. 4 Changes in grooming behavior with illumination. a Duration of grooming sequences decreased with increasing light intensity; grey dashed lines separate experimental groups; WSRT was used for data comparison. b The relative duration of average grooming sequences during S2; the values were obtained by dividing average durations of grooming sequences in S2 by those during S1 for each experimental group. c The number of grooming sequences; paired $t$-test was used for data comparison. d Correlation between the total grooming and immobility behavior durations during S2; the numbers were obtained from Figure 3 plots as indicated by the inset; color coding is as in panels a-c. e Relative durations of antennal grooming and grooming of mid and hind legs

Fig. 5 Dependencies of changes in behavior on light intensity. Fractional durations of the four behaviors during session 2 are plotted against the photon fluxes during light stimulation; "dark" refers to dark red light illumination in S2 experiments in control; "locom." stands for "locomotion". The duration values are the same as in Fig. 3

Fig. 6 Responses of UV- and green-sensitive photoreceptors. Action spectra were obtained by applying $20 \mathrm{~ms}$ isoquantal flashes of light (arrows) from 14 LEDs. a Responses of a typical UVsensitive photoreceptor are shown for first seven LEDs; the superimposed $1 \mathrm{~s}$ voltage response traces are shifted relative each other in $100 \mathrm{~ms}$ steps for presentation purposes; the numbers stand for LED wavelengths. b Responses of a typical green-sensitive photoreceptor. c Dependencies of voltage transient amplitudes ("relative responses") and spectral sensitivities of the photoreceptors from panels a and $\mathbf{b}$ on the peak LED wavelength; spectral sensitivities were calculated for the on the basis of the previously published control voltage response ranges (Saari et al. 2017) 
Figure 1
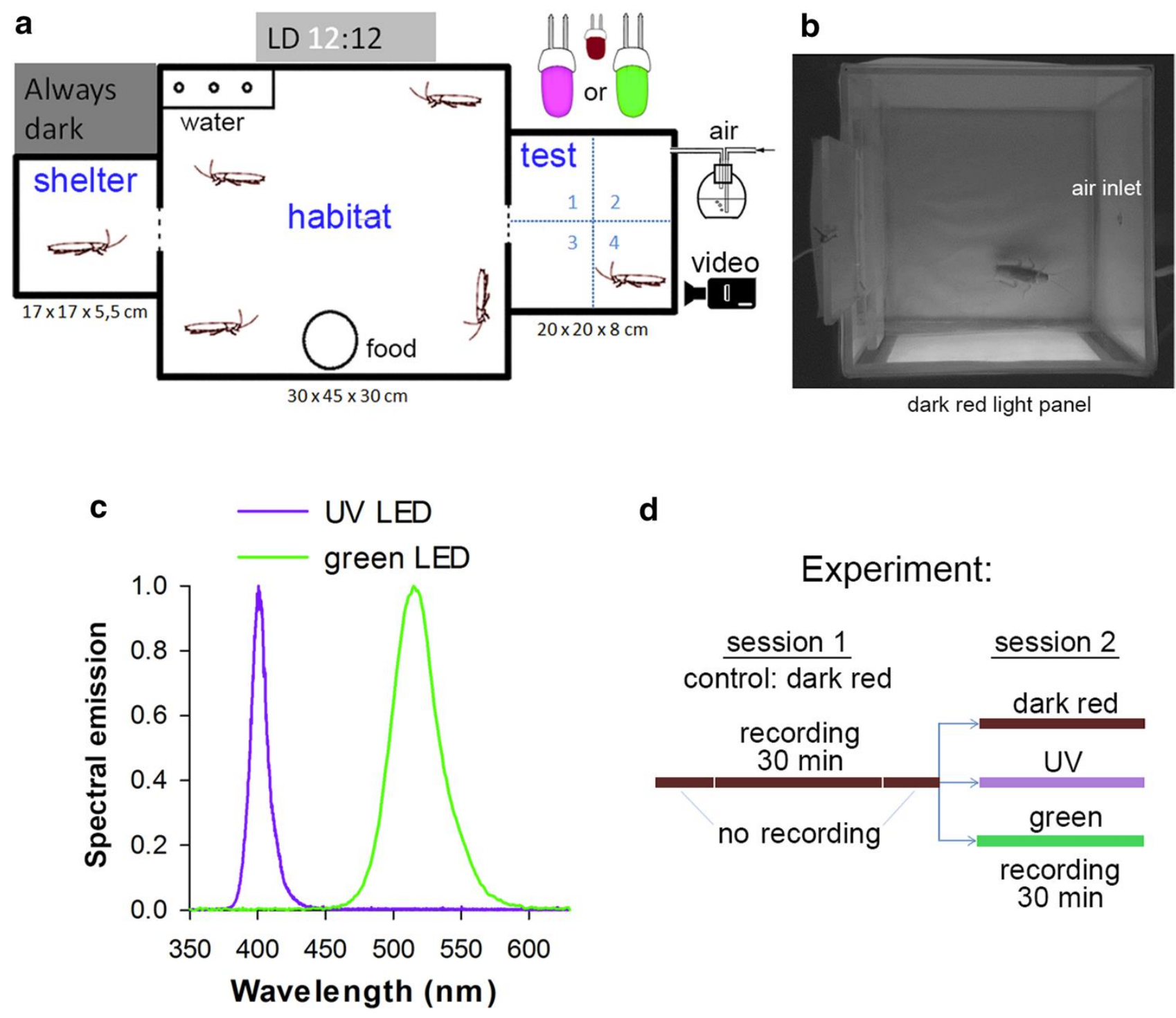

d
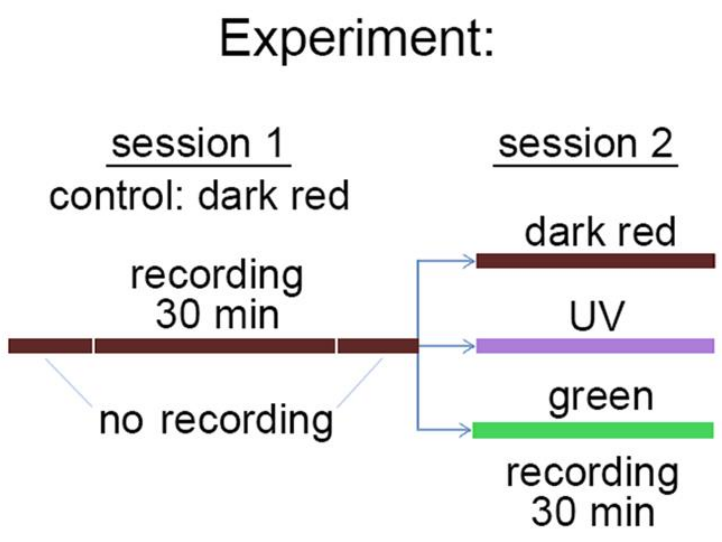
Figure 2
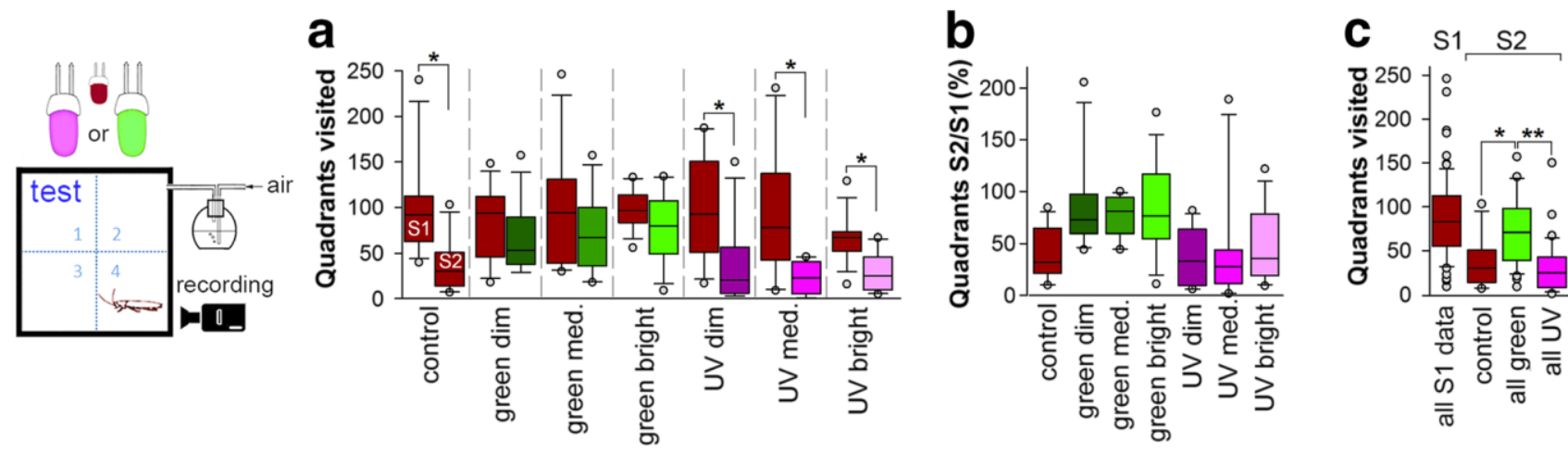
Figure 3

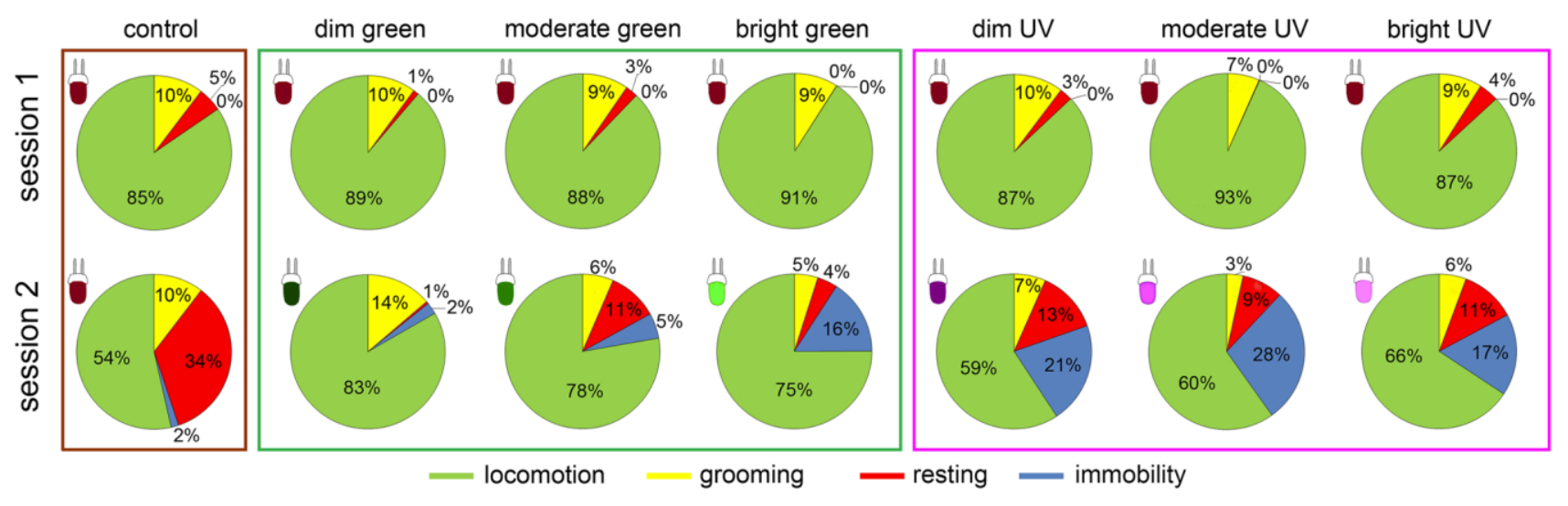


Figure 4
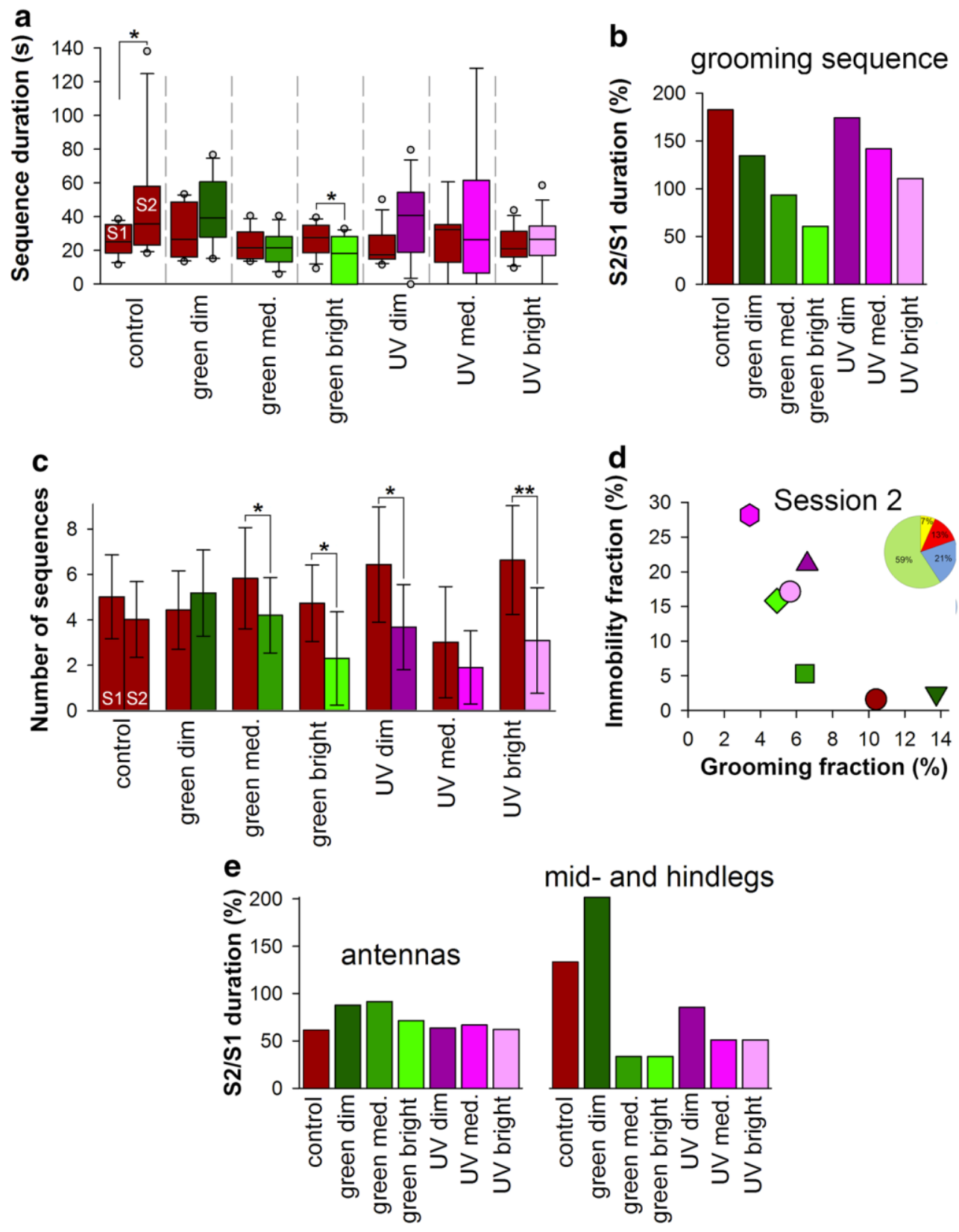
Figure 5
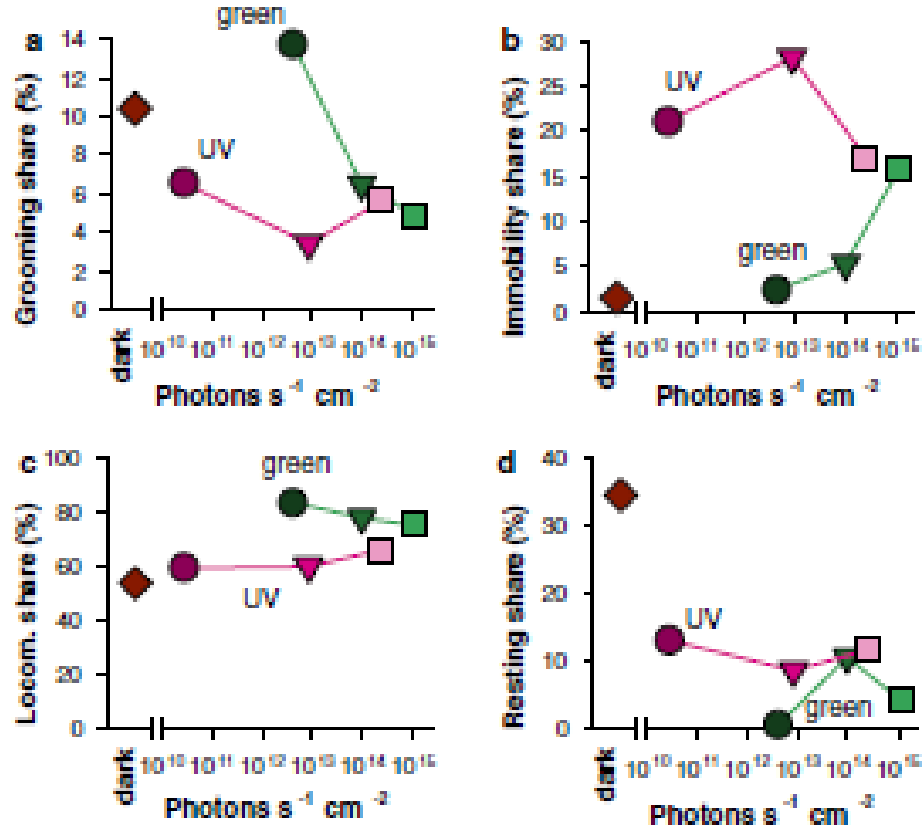
Figure 6
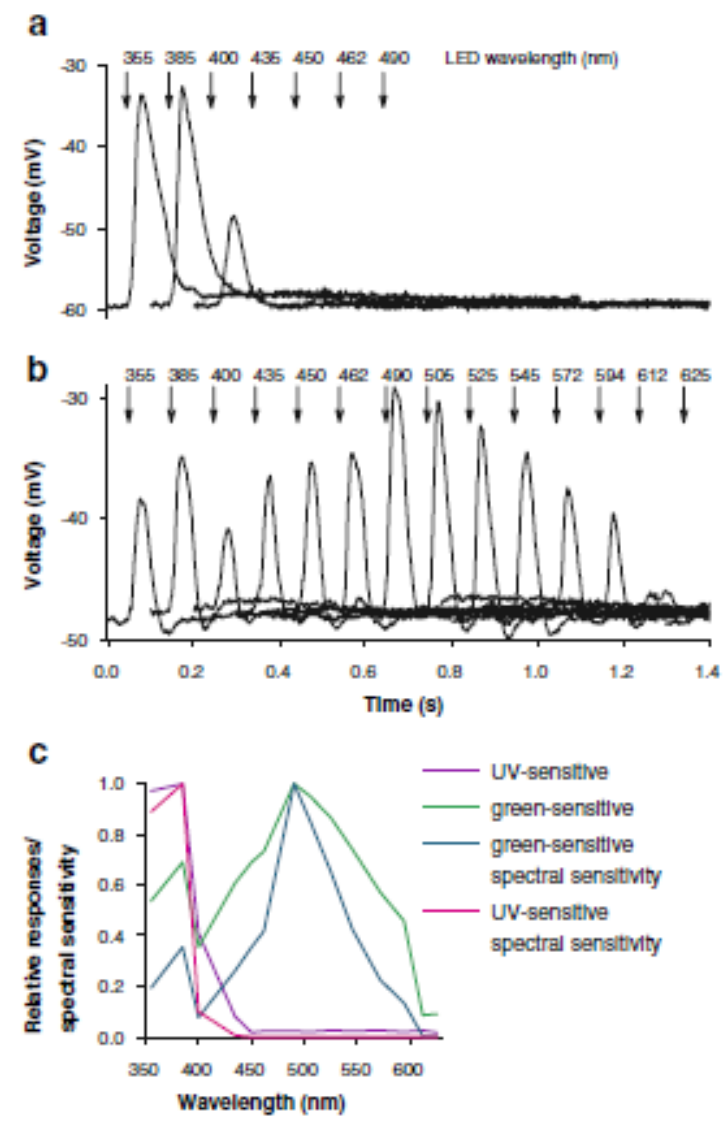